\title{
Rare chondroblastoma of the 6th left rib, video- assisted thoracoscopy resected: one case report and literature review
}

\section{Yonghui Wu}

Depatement of cardiothoracic surgery Third Affiliated Hospital of Sun Yat-Sen University Jiexia Guan

Department of pathology Third Affiliated Hospital of Sun Yat-Sen University

\section{Kai Zhang}

Department of cardiothoracic surgery Third Affiliated Hospital of Sun Yat-Sen University

Huiguo Chen

Department of cardiothoracic surgery Third Affiliated Hospital of Sun Yat-Sen University

Weibin Wu

Department of cardiothoracic surgery Third Affiliated Hospital of Sun Yat-Sen University

Jian Zhang ( $\nabla$ sumszhangjian@163.com )

Third Affiliated Hospital of Sun Yat-Sen University

\section{Case report}

Keywords: Rib tumor, Chondroblastoma, Aggressive tumor, VATS, Case report

Posted Date: April 19th, 2021

DOI: https://doi.org/10.21203/rs.3.rs-370107/v1

License: (9) (i) This work is licensed under a Creative Commons Attribution 4.0 International License.

Read Full License

Version of Record: A version of this preprint was published at Journal of Cardiothoracic Surgery on July 7th, 2021. See the published version at https://doi.org/10.1186/s13019-021-01572-1. 


\section{Abstract}

Introduction: Chondroblastoma is a rare, benign locally but aggressive bone tumor. It accounts for $<1 \%$ of primary bony tumors, and mostly arises from long bones; the rib chondroblastoma is especial rare. Due to its rarity, there are no definitive or standard treatment guidelines.

Case presentation: A case of a 24-year-old male with a chondroblastoma located on the 6th posterior left rib. Computed tomography (CT) demonstrated a rib tumor that was a well-defined oval lesion of $20 \mathrm{~mm} \times$ $18 \mathrm{~mm}$, with lytic bone destruction. The imaging first diagnosis was Langerhans cell histiocytosis (LCH), a giant cell tumor, or other type of neoplasm. The whole tumor and a part of the rib were resected by video-assisted thoracoscopy surgery (VATS). Pathological and immunohistochemical (IHC) examination made a diagnosis of chondroblastoma. Compared with traditional open thoracic surgery, VATS can achieve the same effects and cause less injury to patient. No postoperative adjuvant therapy was given, and had followed 23 months after surgery, there was no recurrence or metastasis.

Conclusion: Chondroblastoma has a risk of recurrence and metastasis, surgery plays a role in the treatment of chondroblastoma, and VATS can achieve the same outcome as open thoracic surgery with less pain and lung function. Close follow-up is needed postoperative.

\section{Background}

Chondroblastoma was first described as a "giant cell tumor with calcifications" by Kolodny in 1927, Ewing described it as a "calcifying giant cell tumor" in 1928, and Codman described it as an "epiphyseal chondromatous giant cell tumor" in 1931 [1]. Chondroblastoma is a rare, benign locally aggressive bone tumor that accounts for $<1 \%$ of primary bony tumors [2]. It characteristically occurs in the epiphyses and secondary ossification centers of long bones, particularly the femur and tibia. The chondroblastoma of the rib is very rare.

The World Health Organization (WHO) has defined chondroblastoma as originating in the epiphyses of skeletally immature persons [3]. The tumor can affect people of all ages, but usually occurs in adolescents and young adults, and men are more commonly affected than women with a male to female ratio of 2:1 [3-5]. About $40 \%$ of tumors occur in adolescents. Chondroblastoma is associated with recurrence and pulmonary metastases $[6,7]$. Herein, we present the case of a 24-year-old male with a chondroblastoma of the 6th left rib that was resected by VATS. Following 23 months after surgery the patient has no signs of recurrence or metastasis.

\section{Case Presentation}

A 24-year-old male was complained of intermediate dry cough for one month. He denied any history of fever, weight loss, chest pain. Chest CT showed a tumor located on the posterior 6th left rib, which measured about $20 \mathrm{~mm} \times 18 \mathrm{~mm}$. An area of local bony destruction with conserved cortex was noted, and the margins were delineated with some ossifying matrix. Enhanced CT revealed non-uniform 
enhancement. There was no evidence of extension to the adjacent soft tissue. The imaging first diagnosis was LCH, a giant cell tumor, or other type of neoplasm. (Fig. 1)

His family medical history was negative for any type of malignancy. On physical examination, the tumor was not palpable and no pain by pressing on the chest wall. The overlying skin was normal, and no sensory deficit. Laboratory testing results, including tumor markers, complete blood cell count and erythrocyte sedimentation rate were within the normal range. The derivation purified protein skin test and tuberculosis test were negative.

VATS was used to resect the tumor and partial broken rib. It was observed to be present on the posterior side of the 6th left rib, the margins were well-defined, and no evidence of adjacent soft tissue invasion was noted. The tumor was resected from the rib, and broken cortical bone was noted. Frozen section pathological examination demonstrated a benign or a low-grade malignant bone tumor. Thus, the broken area of the rib including a margin of at least $10 \mathrm{~mm}$ of normal rib and adjacent partial intercostal muscle were resected. No reconstruction was required for the rib deficit. (Fig. 2)

Histopathological examination of hematoxylin and eosin (HE) stained revealed typical polygonal-shaped chondroblasts and osteoclast-like giant cells. The chondroblasts were large and closely packed with a central, characteristically translucent cytoplasm and grooved nucleus. (Fig. 3)

IHC examination made a diagnosis of chondroblastoma. (Fig. 4)

There were no intraoperative or postoperative complications, and the patient was discharged after five days. Patient described only minimal pain, and had only 2 small incisions on the chest wall. There was little impact on lung function, and a shorten chest drainage tube time and hospital stay. (Fig. 5)

Radical resection and no adjuvant therapy were given postoperatively. The patient was followed every 3 months with chest CT or chest radiograph and blood tests within the first year and every 4 months within the second year. The patient has no chest pain, chest tightness, shortness of breath, no abnormal breathing on the chest wall and the lung function is normal. About 23 months follow-up there is no evidence of recurrence or lung metastases.

A timeline showed the whole medical procedure of this case. 


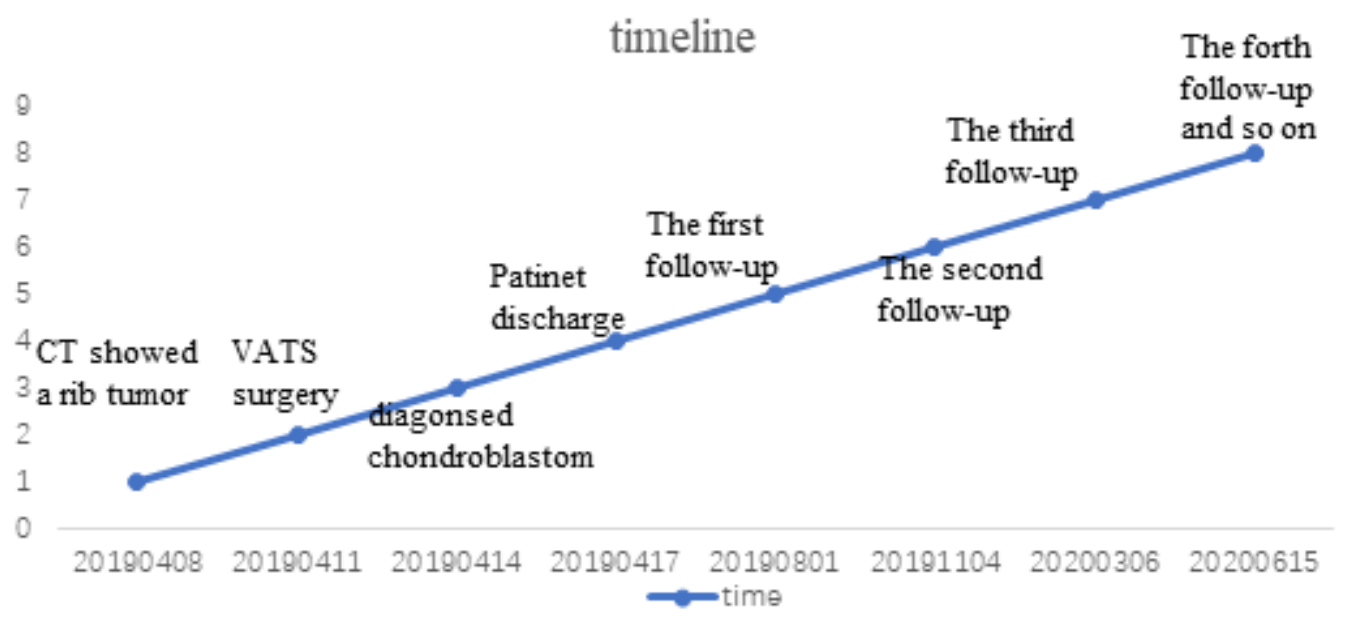

\section{Discussion}

Although only few chondroblastoma of the rib has been reported in the English literature, there are no definitive or standard treatment guidelines. Only rare cases reported that described the diagnosis, radiographic and histological findings, treatment, and follow-up.

Local pain is the most common symptom of chondroblastoma. Other signs and symptoms include in swelling, effusion or pathological fractures on rib, physical examination may show swelling and local tenderness $[3,5]$. The case did not describe any pain or discomfort associated with the tumor, and we believe this was because the tumor was small and there was no invasion of adjacent tissues or intercostal nerve involvement.

CT and Magnetic resonance imaging (MRI) are helpful in making a diagnosis [8]. CT may reveal cortical thinning, cortical expansion with erosion, a periosteal reaction, and calcifications in center of the tumor; unusual radiographic changes may also be seen [1]. It can provide the tumor size and identify extension of the lesion into cortical bone, and it is helpful for planning surgery. MRI can identify a soft tissue mass and medullary invasion, and the tumor presents as low signals on T1-weighted images, and with less inhomogeneity and high signals on T2-weighted images. MRI is useful for determining the extent of the tumor, and identifying pathological fractures within the lesions. On contrast-enhanced MRI, the tumors exhibit lobular, marginal, and septal enhancement [9].

Chondroblastoma is relatively benign tumors, and microscopic examination reveals typical polygonalshaped chondroblasts and osteoclast-like giant cells. HE shows chondroblasts that are large and closely packed with a central, characteristically translucent cytoplasm and grooved nucleus. In general, chondroblasts and osteoclast-like giant-type cells will be observed [3]. Scattered throughout are small, nucleated giant cells and islands of more mature cartilage. Mitotic features can be present, but are sparse among the generally well-defined nuclei. Calcifications are typically present, and have an intercellular distribution with a "chicken wire" or "picket fence" appearance [2].The chicken-wire pattern of calcification is helpful in making a firm diagnosis. However, it is not present in the majority of lesions making it a 
secondary diagnostic criterion. IHC staining for $\mathrm{S} 100$ or $\mathrm{K} 36 \mathrm{M}$ is positive in chondroblastoma, and is a useful ancillary method for making a diagnosis of chondroblastoma $[9,10]$.

Chondroblastoma belongs to a benign tumor, locally aggressive tumor surgery plays a role in all treatments. Some studies also suggested that chondroblastoma of flat bones are more aggressive than those in long bones [11]. Recurrence after surgery is reported to occur in $10-36 \%$ of cases [1, 12], and recurrence rates approaching $20 \%$ have been reported even with complete resection [5]. In our case we did not perform traditional open thoracic surgery instead of VATS resecting the tumor and broken rib with a wire saw. Intraoperative frozen section pathological examination confirmed that the margins of the resected specimen were negative. Compared with the traditional surgery, VATS is minimally invasive and only requires two small incisions, has little or even no impact on lung function, and is associated with a reduced requirement of analgesics, shorter chest drainage tube time, and shorter hospital stay postoperative. In addition, postoperative chest wall numbness is less than that with traditional open thoracic surgery. Earlier reports have advocated curettage for the removal of the tumor [13], but increased recurrence rates have been reported when only curettage is performed for the aggressive tumors [11]. Complete resection is necessary for aggressive tumors (as in our patient).

To reduce the risk of recurrence and metastasis, close and regular follow-up is necessary postoperative. Imaging studies are useful for early diagnosis of tumor recurrence. Follow-up can include physical examination and chest CT every 3 months, and bronchoscopy, abdominal ultrasound, brain MRI, and a bone scan every 6 months for the first 3 years is necessary, then every year to 5 years postoperatively [13]. Close and extensive follow-up may improve the outcomes of patients through detection of asymptomatic recurrences and metastasis.

Radiotherapy is a treatment option for patients who are poor surgical candidates, and for patients with recurrent or unresectable disease, and prevent the recurrence, but which is not a standard method treatment[10]. Radiotherapy is not recommended after complete resection, due to the possibility of radiation-induced chondrosarcoma [6]. At present, there is no role for chemotherapy in the management of chondroblastoma [8].

Metastasis most frequently involves the lungs, tends to occur at the time of primary tumor recurrence, and may be present at the time the bone lesion is identified. Lung metastases are clinically nonprogressive, and can be treated by limited surgical resection or simple observation.

The primary limitation of this report is the short follow-up time; longer follow-up is necessary to evaluate the efficacy of treatment.

\section{Conclusions}

Chondroblastoma is a benign, locally aggressive tumor that occurs on the rib very rare. The primary treatment is surgical resection for the local lesion. Compared with traditional open thoracic surgery, VATS can achieve the same effect and allows a faster recovery. Radiotherapy is a treatment option for poor 
surgical candidates, and for patients with recurrent or unresectable disease. Regular follow-up is necessary to identify recurrence and metastases early and provide appropriate treatment.

\section{Abbreviations}

CT: Computed tomography; LCH: Langerhans cell histiocytosis; VATS: video-assisted thoracoscopy surgery; IHC: immunohistochemical; WHO: World Health Organization; HE staining: hematoxylin and eosin staining; MRI: Magnetic resonance imaging.

\section{Declarations}

Acknowledgements

No.

\section{Authors' contributions}

WYH and GJX drafted the manuscript, acquired the images and pathological pictures. ZJ revised the manuscript. WYH, CHG and ZJ finished this operation. All authors read and approved the final manuscript.

\section{Funding}

No.

\section{Availability of data and materials}

Not applicant.

\section{Ethics approval and consent to participate}

The ethics committee of The Third Affiliated Hospital of Sun Yat-Sen University approved the study.

\section{Consent for publication}

The patient provided written informed consent.

All presentations of case reports have consent to publish.

\section{Competing interests}

We declare that we do not have any commercial or financial interest that is in connection with the work submitted.

Author details 
${ }^{1}$ Department of Cardiothoracic Surgery, the Third Affiliated Hospital of Sun Yat-Sen University, No. 600 Tianhe Road, Guangzhou, China.

${ }^{2}$ Department of Pathology, the Third Affiliated Hospital of Sun Yat-Sen University, No. 600 Tianhe Road, Guangzhou, China.

\section{References}

1. Lambert J, Verstraeten T, Mermuys K: Chondroblastoma: An Unusual Cause Of Shoulder Pain In Adolescence. J Belg Soc Radiol 2016, 100(1):16.

2. Li XN, Peng ZG, Zhao JP, Zhang ZK: Chondroblastoma of the navicular bone. Iran J Radio/ 2014, 11(4):e10848.

3. Akhtar K, Qadri S, Sen Ray P, Sherwani RK: Cytological diagnosis of chondroblastoma: diagnostic challenge for the cytopathologist. BMJ Case Rep 2014, 2014.

4. Lang Y, Yu Q, Liu Y, Yang L: Chondroblastoma of the patella with pathological fracture in an adolescent: a case report. World J Surg Oncol 2019, 17(1):218.

5. Zekry KM, Yamamoto N, Hayashi K, Takeuchi A, Araki Y, Alkhooly AZA, Abd-Elfattah AS, Fouly EH, Elsaid ANS, Tsuchiya H: Surgical treatment of chondroblastoma using extended intralesional curettage with phenol as a local adjuvant. J Orthop Surg (Hong Kong) 2019, 27(3):2309499019861031.

6. Calvert N, Wood D: Use of denosumab in recurrent chondroblastoma of the squamous temporal bone: a case report. Clin Case Rep 2017, 5(4):411-413.

7. Barman S, Diwaker P, Bansal D, Wadhwa N, Singh G: Aneurysmal Bone Cyst: An Uncommon Secondary Event in Calcaneal Chondroblastoma. J Clin Diagn Res 2016, 10(6):ED14-16.

8. Reid L, Wong D, Lyons B: Chondroblastoma of the Temporal Bone: A Case Series, Review, and Suggested Management Strategy. Skull Base Reports 2011, 1(02):071-082.

9. Yang Z, Yang N, Ou Q, Xiang Y, Jiang T, Wu X, Bao H, Tong X, Wang X, Shao YW et al: Investigating Novel Resistance Mechanisms to Third-Generation EGFR Tyrosine Kinase Inhibitor Osimertinib in Non-Small Cell Lung Cancer Patients. Clin Cancer Res 2018, 24(13):3097-3107.

10. Liu J, Ahmadpour A, Bewley AF, Lechpammer M, Bobinski M, Shahlaie K: Chondroblastoma of the Clivus: Case Report and Review. J Neurol Surg Rep 2015, 76(2):e258-264.

11. Arikan M, Togral G, Yildirim A, Irkkan C: A rare case of chondroblastoma of the acromion. Acta Orthop Traumatol Turc 2016, 50(6):691-693.

12. Laitinen MK, Stevenson JD, Evans S, Abudu A, Sumathi V, Jeys LM, Parry MC: Chondroblastoma in pelvis and extremities- a signle centre study of 177 cases. J Bone Oncol 2019, 17:100248.

13. Brandolini J, Bertolaccini L, Pardolesi A, Salvi M, Valli M, Solli P: Chondroblastoma of the rib in a 47year-old man: a case report with a systematic review of literature. Journal of Thoracic Disease 2017, 9(10):E907-E911. 


\section{Figures}
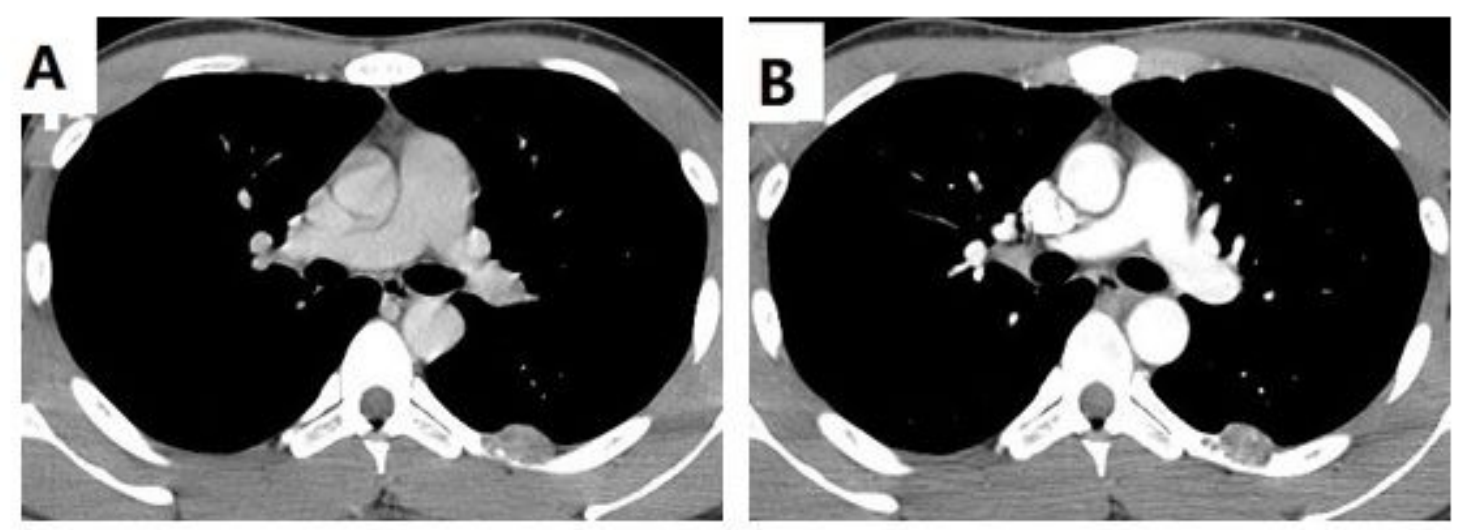

\section{Figure 1}

Chest CT showed that there was a tumor located the 6th posterior left rib and bony cortex erosion $(A)$. Enhanced CT could saw the tumor non-uniformity enhancement (B).

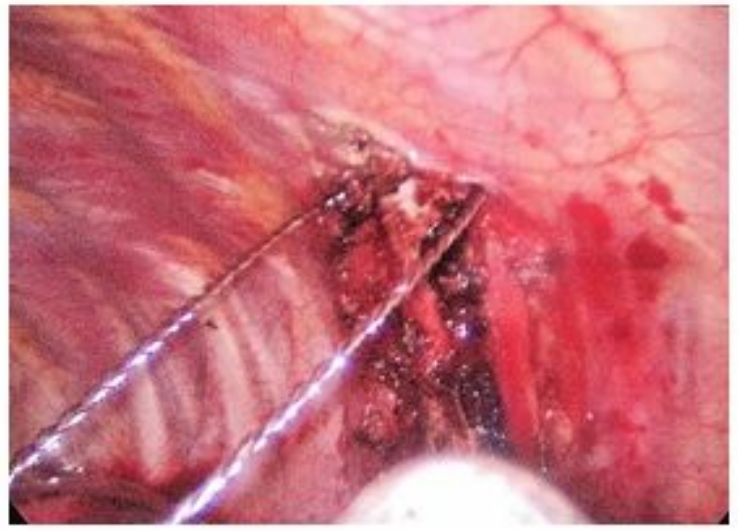

Figure 2

VATS showed that a thin and broken periosteal shell was exposed, the surrounding soft tissue wasn't invaded by tumor. Removed the tumor and broken rib with wire saw.
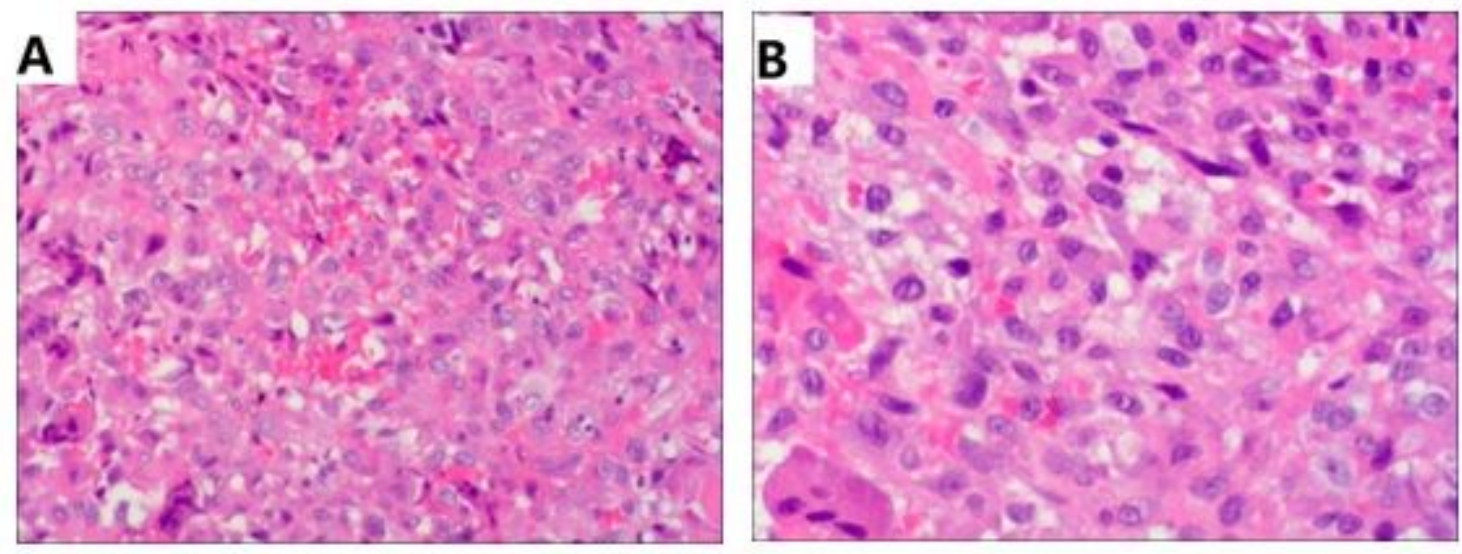


\section{Figure 3}

H\&E staining showed that tumor was filled with chondroblasts which were large and closely packed, characteristically translucent cyto plasm (A, H\&E x 100) and a little grooved nucleus (B, H\&E x 200).
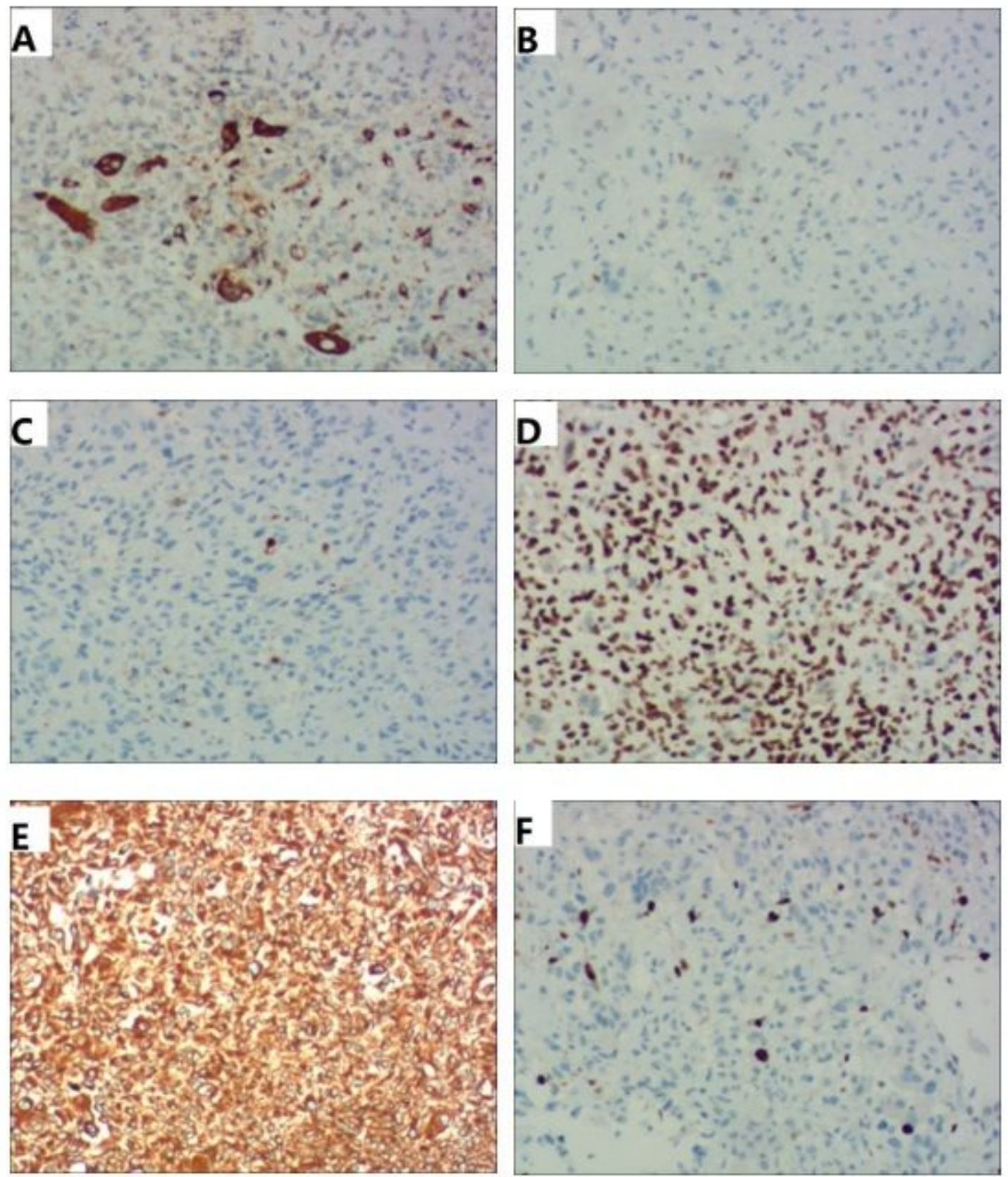

\section{Figure 4}

IHC examination (all staining x 100): (A):CD68 positive; (B): p63 weak positive;(C): S-100 protein negative; (D): SATB positive;(E): VIM positive; (F)Ki-67 about $10 \%$. 


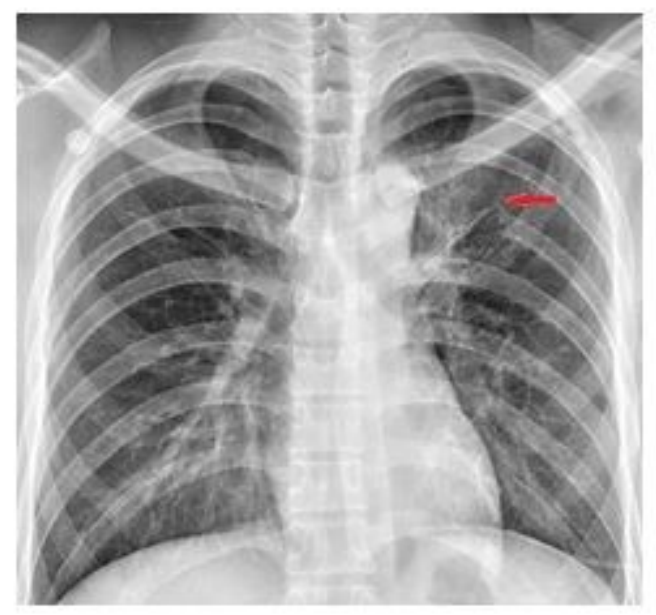

Figure 5

Chest X-ray showed that there was the partial 6th left posterior rib defects and no reconstruction.

\section{Supplementary Files}

This is a list of supplementary files associated with this preprint. Click to download.

- CAREchecklistEnglish2013.pdf 